limited to high-risk patients receiving ventilation. Furthermore, mortality day $0-30$ post discharge also fell.

\begin{tabular}{lllll}
\hline Abstract P190 Table 1 & & & & \\
\hline Inpatient Mortality & All patients & $223 / 3943$ & $90 / 2348$ & 0.0012 \\
& $(\%)$ & $(5.66)$ & $(3.83)$ & \\
& Ventilated (\%) & $71 / 540$ & $32 / 346$ & 0.086 \\
& & $(13.15)$ & $(9.25)$ & \\
& Not ventilated & $152 / 3403$ & $58 / 2002$ & 0.0035 \\
& $(\%)$ & $(4.47)$ & $(2.90)$ & \\
Inpatient+30 day combined & All patients & $309 / 3943$ & $123 / 2348$ & $<0.0001$ \\
mortality & $(\%)$ & $(7.84)$ & $(5.24)$ & \\
& Ventilated (\%) & $98 / 540$ & $36 / 346$ & 0.0015 \\
& & $(18.15)$ & $(10.40)$ & \\
& Not ventilated & $211 / 3403$ & $87 / 2002$ & 0.0037 \\
& $(\%)$ & $(6.20)$ & $(4.35)$ & \\
\hline
\end{tabular}

\section{P191 GENDER DIFFERENCES IN COPD EXACERBATIONS: ANALYSIS FROM THE CPRD DATABASE}

${ }^{1} \mathrm{D}$ Stolz, ${ }^{1}{ }^{2} \mathrm{~K}$ Kostikas, ${ }^{2} \mathrm{E}$ Loefroth, ${ }^{3} \mathrm{R}$ Fogel, ${ }^{2} \mathrm{~A}$ Clemens, ${ }^{2} \mathrm{FS}$ Gutzwiller, ${ }^{4} \mathrm{~V}$ Conti, ${ }^{3} \mathrm{H}$ Cao. ${ }^{1}$ Pneumology Department, University Hospital, Basel, Switzerland; ${ }^{2}$ Novartis Pharma $A G$, Basel Switzerland; ${ }^{3}$ Novartis Pharmaceuticals Corporation, East Hanover, NJ, US; ${ }^{4}$ Novartis Ireland Limited, Dublin, Ireland

\subsection{6/thoraxjnl-2017-210983.333}

Introduction and Objective In recent years accumulating evidence supports gender differences in COPD, suggesting a steady increase in COPD prevalence and mortality rates in women. In this analysis we evaluated gender differences in COPD exacerbations in a cohort of COPD patients from the Clinical Practice Research Datalink (CPRD), a general practice electronic primary medical care records database in the UK.

Methods This is a retrospective cohort study comparing women and men with an incident diagnosis of COPD, using secondary data from the linkage between the CPRD and the Hospital Episode Statistics (HES) databases. The study period was between 01 January 2005 and 28 February 2016; patients with an incident diagnosis of COPD between 01 January 2010 and 28 February 2015 were included in this study.

Results A cohort of 22,429 COPD patients (48\% women) with an incident diagnosis of COPD was identified. At diagnosis, women were younger, more often current or non-smokers and had lower BMI, better lung function (as expressed by $\mathrm{FEV}_{1} \%$ predicted), worse mMRC dyspnea scale scores and lower blood eosinophils. Women also had a higher prevalence of asthma, anxiety, depression and osteoporosis, whereas men had more often cardiovascular comorbidities (myocardial infarction, heart failure and atrial fibrillation). The risk of first moderate or severe exacerbation was $17 \%$ greater in women than in men (adjusted HR, 1.17; 95\% CI, 1.12 to 1.23), with a median time to first exacerbation of 504 days for women and 637 days for men. These gender differences were more prominent in patients aged 40-64 years and in those with moderate-to-severe airflow obstruction $\left(30 \% \leq \mathrm{FEV}_{1}<80 \%\right.$ predicted). Women also had a greater rate of moderate or severe exacerbations at year 1 (adjusted RR, 1.15; 95\% CI, 1.07 to 1.23 ), year 2 (adjusted RR, 1.14; 95\% CI, 1.08 to 1.21) and year 3 (adjusted RR, 1.14; 95\% CI, 1.08 to 1.20) of follow-up.

Conclusions Despite evidence for milder disease at the time of COPD diagnosis, women were at greater risk of COPD exacerbations than men, especially at younger ages. These Results highlight the unmet need for appropriate identification and management of women with COPD in clinical practice.

\section{P192 FUNCTIONAL RESPIRATORY IMAGING (FRI) AND LUNG FUNCTION ASSESSMENT OF GLYCOPYRRONIUM/ FORMOTEROL FUMARATE DIHYDRATE FIXED-DOSE COMBINATION DELIVERED USING INNOVATIVE CO- SUSPENSION DELIVERY TECHNOLOGY (GFF MDI) IN COPD}

${ }^{1} \mathrm{~W}$ De Backer, ${ }^{2} \mathrm{~J}$ De Backer, ${ }^{2} \mathrm{~W}$ Vos, ${ }^{2}$ Verlinden, ${ }^{3} \mathrm{~S}$ Dwivedi, ${ }^{4} \mathrm{~S}$ Siddiqui, ${ }^{5} \mathrm{M}$ Jenkins, ${ }^{4,6} \mathrm{C}$ Reisner, ${ }^{4} \mathrm{U}$ Martin. ${ }^{1}$ University of Antwerp, Antwerp, Belgium; ${ }^{2}$ FLUIDDA, Kontich, Belgium; ${ }^{3}$ Pearl Therapeutics Inc., Redwood City, US; ${ }^{4}$ AstraZeneca, Gaithersburg, US; ${ }^{5}$ AstraZeneca, Cambridge, UK; ${ }^{6}$ Pearl Therapeutics Inc., Morristown, Gaithersburg; US

\subsection{6/thoraxinl-2017-210983.334}

Introduction and Objectives FRI has been used to provide a detailed view of overall and local changes in airway resistance and lung volume, in addition to spirometry and body plethysmography. The aim of this study was to assess changes in specific image-based airway volume (siVaw) and resistance (siRaw) in patients with moderate-to-severe COPD after administration of GFF vs placebo.

Methods In this double-blind, 2 week dosing, Phase III crossover study (NCT02643082), 20 patients (40-80 years) received twice daily GFF MDI $14.4 / 10 \mu \mathrm{g}$ (equivalent to glycopyrrolate/formoterol fumarate $18 / 9.6 \mu \mathrm{g}$ ) and placebo MDI. Primary endpoints were siVaw and siRaw at Day 15. Additional outcomes included spirometry, plethysmography and safety.

Results On Day 15, GFF MDI led to an estimated $75 \%$ increase in siVaw and $71 \%$ decrease in siRaw relative to placebo MDI (both $\mathrm{p}<0.0001$; Table), accompanied by post-dose differences of $443 \mathrm{~mL}$ in $\mathrm{FEV}_{1}$ and $454 \mathrm{~mL}$ in IC in change from baseline for GFF MDI vs placebo MDI (both $\mathrm{p}<0.001$ ). The safety profile was consistent with the drug class with no unexpected safety findings.

Conclusions The dual bronchodilator GFF MDI demonstrated significant benefits on FRI-based airway volume and resistance in the lungs of COPD patients. Benefits were associated with important improvements in $\mathrm{FEV}_{1}$, IC and hyperinflation. 